\title{
Type Ib Supernova 2007uy - a multiwavelegth perspective
}

\author{
Rupak Roy and Brijesh Kumar
}

Aryabhatta research institute of observational sciences (ARIES), Nainital, India

\begin{abstract}
We present the results from a comprehensive analysis of a Type Ib supernova (SN) 2007uy in a nearby galaxy NGC 2770 ( $\sim 30 \mathrm{Mpc})$, using data from space-based Swift/UVOT, along with ground-based observations at visible, infrared and radio wave bands.
\end{abstract}

Keywords. (Stars:) Supernovae: individual (SN 2007uy); techniques: photometric, spectroscopic; radio continuum: Supernovae

\section{Introduction}

The stripped-envelope supernovae of Type Ibc are rare and the detailed characteristics of explosion as well as the nature of progenitors have been studied only for a very few such events. Pre-SN imaging revealed that majority of Type II events are originated from supergiants. Recent investigation shows that progenitors of Type Ibc are probably Wolf Rayet stars, which are visually faint and hence challenging to get detected in comparison to low mass progenitors of Type II events (Yoon et al. 2012).

The supernova (SN) 2007uy is a type Ib event discovered on 31.7 UT December, 2007 by Yoji Hirose in the nearby spiral galaxy NGC 2770 (Nakano et al. 2008). Blondin \& Calkins (2008), took the first spectrum of this event with FAST on the 1.5-m reflector at F. L. Whipple Observatory and its comparison with that of template type Ibc SNe spectra using the SNID tool (Blondin \& Tonry 2007) revealed that SN 2007uy is young and the spectrum is similar to SN 2004gq at roughly one week before maximum.

\section{Observations, data analysis and results}

Optical photometric observation was performed using ground-based 1-m Sampurnanand Telescope (ST), 2.5-m NOT and Space-based Swift/UVOT. The NUV photometry was performed using Swift/UVOT, whereas NIR observations were conducted from 3.8-m UKIRT. Archival data from VLA have been used for radio follow-up. The spectroscopic data were acquired from 2.5-m NOT, 3.6-m NTT, 8.2-m VLT and 6.5-m MMT. For optical data, we performed template subtraction technique and applied standard stellar psf photometry (DAOPHOT), to get the calibrated magnitudes of the SN event.

Comparison of UVOIR light curves of SN 2007uy with other Type Ibc events shows that the supernova was discovered within a week after its explosion. We found that the reddening due to host is $\mathrm{E}(\mathrm{B}-\mathrm{V}) \sim 0.69 \mathrm{mag}$, which is substantially higher from the Galactic reddening (0.02 mag) along the line of sight, having contribution mainly from the highly inclined host galaxy. The UVOIR bolometric light curve has been computed and compared with different Type Ibc events (see left panel of fig. 1) and it is as luminous as GRB associated event SN 1998bw. After modeling the UVOIR bolometric light curve, we estimated the ejected mass $\sim 2 \mathrm{M}_{\odot}$, while amount of radioactive ${ }^{56} \mathrm{Ni}$ produced during this explosion is $\sim 0.4 \mathrm{M}_{\odot}$. 

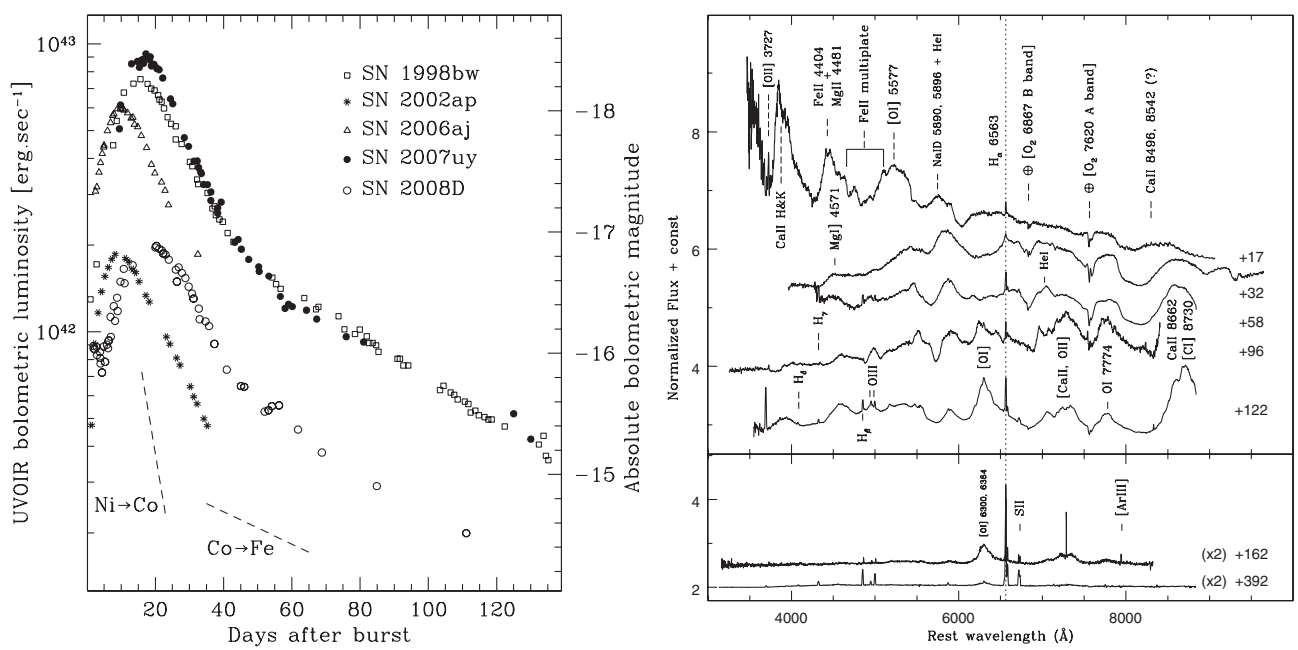

Figure 1. Left Panel: The UVOIR bolometric light curve of SN 2007uy. Comparison with other Type Ibc events. Right Panel: Spectroscopic evolution of SN 2007uy. All the spectra have been normalized with respect to the peak flux of the underling $\mathrm{H}_{\alpha}$ feature and a constant offset has been applied to present them clearly. The $+162 \mathrm{~d}$ and $+392 \mathrm{~d}$ spectra have been multiplied by a factor of 2 to enlarge several tiny features. The dotted vertical line represents the position of $\mathrm{H}_{\alpha}$ and confirms the wavelength calibration within the limits of the spectral resolution.

The event was monitored spectroscopically over an year using data from several 2 to $10-\mathrm{m}$ class telescopes over the globe. The spectral evolution of the event is presented in right panel of fig. 1 . The asymmetric evolution of different line profiles are clearly visible. The features are highly blended. The measured value of photospheric velocity around the peak is roughly around $15200 \mathrm{~km} \mathrm{~s}^{-1}$. We found that the temporal evolution of line profiles for different species are not similar to each other. This probably implies that there is no major effect of radioactive $\mathrm{Ni}$ on the evolution of different lines, at least for this particular case. The interaction of SN shock with the surrounding CSM generates radio waves. The archival radio data has been reduced, analyzed and modeled to find out several interaction parameters. According to radio data modeling the explosion happen nearly 4 days before the discovery of the event. This result is consistent with spectroscopic study. We measured the mass loss rate of this event during pre-SN phase and it is found to be more than $2.4 \times 10^{-5} \mathrm{M}_{\odot} \mathrm{yr}^{-1}$.

\section{Acknowledgements}

This contribution is based on the work by Roy et al. (2013), which is under review.

\section{References}

Blondin S., Tonry J. L. 2007, ApJ, 6 66, 1024-1047

Blondin S. \& Calkins M. 2008, CBET, 1191, 2

Nakano S., Kadota K., Itagaki K., \& Corelli P. 2008, CBET, 1191, 1

Yoon S. C., Gräfener G., Vink J. S., Kozyreva A., \& Izzard R. G. 2012, A\& A, 544, L11 